\title{
Genetic analysis of parthenogenetic capability and fecundity in Drosophila albomicans
}

\author{
Chia-chen Chang ${ }^{1}$ and Hwei-yu Chang ${ }^{1,2^{*}}$
}

\begin{abstract}
Background: The successful rate of parthenogenesis in Drosophila harvested from natural population was extremely low, which could be effectively improved under selection pressure. Facultative parthenogenesis in Drosophila albomicans may be advantageous for its expansion from sub-tropical to temperate area. Since the understanding of the genetics involved in the capability and fecundity of parthenogenesis is limited, this study aims to preliminarily map the chromosome regions that are preferentially important for parthenogenesis.

Results: Genetic mapping was performed with $F_{2}$ individuals that were parthenogenetically produced by $F_{1}$ from crosses between a parthenogenetic strain KKU119 and a sexual strain \#55.1 of Drosophila albomicans. Among $105 \mathrm{~F}_{2}$, 53.3\% of them had parthenogenetic capability which is highly associated with three markers a28, c4081, and c7198 located near or inside $\ln (2 L) B_{1} D_{5}$. A sexual strain with high $\ln (2 L) B_{1} D_{5}$ heterozygosity originating from Wulai, Taiwan in 1970 was able to perform parthenogenesis. However, the fecundity of those $F_{2}$ varied in a wide range, forming a continuous distribution as expectation of a quantitative trait and was correlated with the number of homozygous markers for all markers on the second chromosome and neo-X chromosome arm.

Conclusions: We have genetically analyzed the capability and fecundity of parthenogenesis in Drosophila albomicans. The former is specifically associated with a limited region in the $B_{1}$ to $D_{5}$ of $2 \mathrm{~L}$ arm where inversion $\ln (2 L) B_{1} D_{5}$ may play certain role for the maintenance of parthenogenesis, whereas the latter is apparently related to several quantitative loci on the second chromosome and neo-X chromosome arm.
\end{abstract}

Keywords: Facultative parthenogenesis; Genetic mapping; Inversion polymorphism

\section{Background}

Hundreds of lineages in the animal kingdom have experienced the evolution from sexual reproduction to parthenogenesis by which females reproduce offspring without mating (Schwander et al. 2010). As compared to sexual reproduction, the advantages of parthenogenesis include theoretically twofold fecundity, given all else is equal (Maynard Smith 1978), and increased potential of colonization in harsh, high latitude and/or altitude, isolated environments or newly invaded marginal habitats, where population size is usually too small for females to find mates (Suomalainen 1950). Alternatively, parthenogenesis may suffer disadvantages for longterm evolution because of the loss of genetic variation and

\footnotetext{
* Correspondence: hwei@ntu.edu.tw

'Department of Entomology, National Taiwan University, Taipei 10617, Taiwan

${ }^{2}$ Research Center for Biodiversity, Academia Sinica, Nankang, Taipei 11529,
} Taiwan accumulation of deleterious mutations (Simon et al. 2003). Facultative parthenogenesis, coexistence of parthenogenesis and sexual reproduction, is apparently the better reproductive strategy than parthenogenesis (Hurst and Peck 1996).

Most parthenogenetic Drosophila species conduct facultative parthenogenesis (Templeton 1983), and hence, they may escape from the accumulation of deleterious mutations and display higher fitness in the condition of low population density. Although the hatchability of unfertilized eggs of parthenogenetic Drosophila harvested from natural population is extremely low, it could be significantly increased under certain selection pressure (Carson 1967; Stalker 1954; Markow 2013). Since productivities were increased with generations under artificial selection, parthenogenesis is considered to be regulated by polygenes. However, if parthenogenesis is controlled by a

\section{Springer}

(c) 2014 Chang and Chang; licensee Springer. This is an Open Access article distributed under the terms of the Creative Commons Attribution License (http://creativecommons.org/licenses/by/4.0), which permits unrestricted use, distribution, and reproduction in any medium, provided the original work is properly credited. 
polygenic system, successful parthenogens will be hard to evolve (Fuyama 1986). A study on the parthenogenetic Drosophila mercatorum reported that the genotypes for incapability of parthenogenesis were quickly eliminated in the first few generations, and the population size varied among different genotypes after several generations of parthenogenesis (Annest and Templeton 1978). Therefore, we consider that there are only a few essential genes for parthenogenetic capability while there are many genes influencing the fecundity (i.e., the number of parthenogenetically produced offspring). The former phenotype is either capable or incapable to perform parthenogenesis, and the latter indicates the parthenogenetic efficiency of genetically different females. Genetic mapping for parthenogenesis conducted on Drosophila ananassae complex with parthenogenetic capability as phenotype indicated a major gene located on its 2L chromosome arm (Matsuda and Tobari 2004). Fuyama (1986) indicated that Drosophila melanogaster can perform gynogenetic reproduction (i.e., females can reproduce by mating with sterile males), which was related to recessive factors located between $T f t$ locus and $n w$ locus on $2 \mathrm{R}$ chromosome arm and $G l$ and $\mathrm{Sb}$ on $3 \mathrm{R}$ chromosome arm.

In the present study, we used Drosophila albomicans, a species invading from sub-tropical areas (i.e., probably Wulai, Taiwan) to the temperate climate zone (Ohsako et al. 1994), as material for genetic mapping. Parthenogenesis of Drosophila albomicans was first reported by Ohsako and Fuyama (1995) in Kiikatsuura, Japan which was the boundary of Drosophila albomicans distribution. Strong natural selection in favor of parthenogenesis may be operated for an adaptation to a cold climate in which population density is low. Parthenogenetic strain was established with $80 \%$ to $90 \%$ of them capable of parthenogenesis and about 11 offspring were reproduced per female from 11th to 30th generation after strain establishment (Ohsako and Fuyama 1995). In order to find a proper sexual strain to cross with this parthenogenetic strain, we checked the parthenogenetic capability of strains. We crossed between the sexual strain and parthenogenetic strain and collected $F_{1}$ virgin females to produce recombinant $F_{2}$ since meiosis was performed during parthenogenesis. Therefore, the $\mathrm{F}_{2}$ offspring were adopted to map the genetic elements for parthenogenetic capability and do genetic analysis for parthenogenetic fecundity. We also discussed the maintenance of parthenogenesis in Drosophila albomicans.

\section{Methods}

\section{Fly strains}

The parthenogenetic Drosophila albomicans strain was provided as a courtesy from Dr. Fuyama. It was established from a virgin female of an isofemale strain (KKU119), which was originally collected from Kiikatsuura, Japan in
1990, and maintained by parthenogenesis ever since (Ohsako and Fuyama 1995). Isofemale sexual strains \#55.1 and \#56.1 were established in 1970 from Hualien and Wulai, Taiwan, respectively. For the chromosomal inversion arrangements, there is no inversion heterozygote on the neo-sex arm or the $2 \mathrm{R}$ arm in all of these three strains but only one inversion type, $\operatorname{In}(2 L) B_{1} D_{5}$, on the $2 \mathrm{~L}$ arm. The strain \#56.1 has about $75 \% \operatorname{In}(2 L)$ $B_{1} D_{5}$ heterozygosity. The $2 \mathrm{~L}$ arms of KKU119 and \#55.1 are homozygous, but they carry different arrangements. Flies were maintained on standard cornmeal medium at $23^{\circ} \mathrm{C}$ under 12:12 h LD cycle. Flies used for crosses were sexed within $8 \mathrm{~h}$ after eclosion.

\section{Parthenogenetic capability}

In order to do proper genetic mapping, we made sure that the chosen sexual strain could not perform parthenogenesis. The parthenogenetic capability of strains \#55.1 and \#56.1 was examined by transferring virgin females to new vials twice a week until 4 weeks. The vials were checked for offspring reproduced in 4 weeks.

\section{Recombinant individuals}

In order to perform the genetic mapping for parthenogenesis through genotype-phenotype association, we generated recombinant individuals with known parthenogenetic phenotypes: capability and fecundity. Recombinant flies were produced through the following cross scheme: KKU119 females were crossed with \#55.1 males, and their $F_{1}$ virgin females were subjected to reproduce $F_{2}$ parthenogenetically. The phenotypes of $\mathrm{F}_{2}$, including parthenogenetic capability and fecundity, were determined by culturing each individual $F_{2}$ in a vial and transferred to a new vial twice a week for consecutive 4 weeks. The parthenogenetic capability is determined by whether or not the $F_{2}$ parthenogenetically reproduces offspring in 4 weeks and the fecundity is the number of $\mathrm{F}_{3}$. Approximate $100 \mathrm{~F}_{2}$ were genotyped using 14 PCR-RFLP markers, 7 on the second chromosome arm and 7 on the neo-X chromosome arm (Table 1). Single-fly genomic DNA was extracted using the Puregene Cell and Tissue DNA Isolation Kit (Gentra Systems, Minneapolis, MN, USA) following the manufacture's protocol.

\section{Statistical analysis}

The association between genotypes and parthenogenetic capability was revealed by Fisher's exact test. Linkage disequilibrium $D^{\prime}$ (Lewontin 1964) values among highly associated markers were calculated to reveal hitchhiking. When the linkage disequilibrium was high, we checked recombinant individuals through Fisher's exact test in order to reveal which of the two markers was more influential. Recombination rates for all markers were also 
Table 1 Marker list with location, primer name, primer sequence, PCR annealing temperature, and restriction enzyme

\begin{tabular}{|c|c|c|c|c|c|}
\hline Location & Primer name & Primer sequence & Annealing temperature $\left({ }^{\circ} \mathrm{C}\right)$ & $\begin{array}{l}\text { Restriction } \\
\text { enzyme }\end{array}$ & Reference \\
\hline \multirow[t]{14}{*}{ Second chromosome } & $\mathrm{a} 28$ & F: GGGGCACACTGATTTATTAAACAA & 57 & Alul & Chang et al. (2008) \\
\hline & & R: TATITAACGCCACAACTTGCAGCAC & & & \\
\hline & a708 & F: GAAAAGGGCGAACAGATAGA & 55 & $x m n l$ & Chang (2011) \\
\hline & & R: AACAGGAACATAGAAATCAC & & & \\
\hline & A185D & F: CAAACGCTCTGGAATAATGG & 55 & Rsal & Chang (2011) \\
\hline & & R: CTCGGGAGTGTGGGGC & & & \\
\hline & c4081 & F: GCCCTGGAACAAAGTGAAAA & 52 & Haelll & This study \\
\hline & & R: AGTCTGCTGCGTATGGTCAA & & & \\
\hline & c5237 & F: TATATGTTCCTCCTGATTGG & 55 & Pstl & Chang (2011) \\
\hline & & R: AAGTTTAAACGCGAACTTIT & & & \\
\hline & c5665 & F: TGAAACATTATTACCGCCTG & 60 & Haelll & This study \\
\hline & & R: GATGACGACGACGATTCC & & & \\
\hline & C7198 & F: GTGGGAAGCACGTTACAT & 57 & Msel & Chang (2011) \\
\hline & & R: CCATGAACAGTCTGAAGTTT & & & \\
\hline \multirow[t]{14}{*}{ neo-X chromosome arm } & a52 & F: TATTCATCGCATTCCACAT & 53 & Haelll & Chang et al. (2008) \\
\hline & & R: GGCTTCCTCAATCAACTG & & & \\
\hline & a386 & F: GTTACGATTACGAAGAGTGC & 51 & $x m n l$ & Chang et al. (2008) \\
\hline & & R: CTGCCGTGCTTATGTGAT & & & \\
\hline & a1185 & F: ATTCTGTCGTTCGTTTTGA & 49 & Styl & Chang et al. (2008) \\
\hline & & R: GATTTCGGCTTACATTATTG & & & \\
\hline & a1350 & F: TACGACCCCGTCAAAGGCTGTG & 52 & Hpall & Chang (2011) \\
\hline & & R: GGCTTGTATGCGATTCTGC & & & \\
\hline & a1953 & F: GCCAACAGCGAGCCTTCT & 56 & Ddel & Chang et al. (2008) \\
\hline & & R: GCGACCCAAGCACGAATC & & & \\
\hline & $c 29$ & F: CTGGGCAAAGAGTGTAGG & 57 & Rsal & Chang et al. (2008) \\
\hline & & R: CAGAAGGAGGGCGAAAA & & & \\
\hline & c3242 & F: TTGAAGCGCAGTTTATGCAC & 62 & Sspl & Chang (2011) \\
\hline & & R: TACAACCACGACCTGGACAA & & & \\
\hline
\end{tabular}

calculated in order to estimate the unknown locations of markers. In addition, the correlation between the parthenogenetic fecundity and the number of KKU119 homozygous markers carried by $\mathrm{F}_{2}$ was done by the analysis of variance (ANOVA) test, and jackknifing was also performed to figure out whether or not there is any key marker for the fecundity.

\section{Results}

Association between markers linked to $\ln (2 L) B_{1} D_{5}$ and parthenogenetic capability

In order to find a proper sexual strain for genetic mapping, we first analyzed the parthenogenetic capability of these two sexual strains \#55.1 and \#56.1. Among 69 virgin females of strain \#56.1, 2 of them (2.9\%) were found to produce offspring by parthenogenesis, whereas none of 72 virgins of strain \#55.1 was observed to do so. Therefore, \#55.1 was used to perform genetic mapping although they carried different chromosomal inversion arrangements. As to parthenogenetic capability, 56 virgin $F_{2}$ could parthenogenetically reproduce offspring in 4 weeks, indicating that it was $53.3 \%(n=105)$ of those $\mathrm{F}_{2}$. Those $\mathrm{F}_{2}$ were genotyped using 14 PCR-RFLP markers, and the genotyping result is shown in Additional file 1. The alleles from KKU119 were assigned as $P$ and those from \#55.1 as $S$. Since heterozygosity (frequency of PS) was very low (i.e., in average $1.5 \%$ ), only homozygotes (i.e., $P P$ and $S S$ ) were included for analysis. Based on haplotypes, recombination rates among seven markers on the second chromosome and those among seven on the neo-X chromosome arm were calculated and summarized, respectively, in Table 2. The locations of two 
Table 2 Recombination rate (\%) of markers on the second chromosome and neo-X chromosome arm

\begin{tabular}{lllllll}
\hline \multicolumn{7}{c}{ Recombination rate (\%) } \\
\hline Second chromosome & a28 & a708 & A185D & c4081 & c5237 & c5665 \\
a708 & 15.4 & & & & & \\
A185D & 44.6 & 56.9 & & & & \\
c4081 & 1.0 & 16.2 & 43.1 & & & \\
c5237 & 33.7 & 39.0 & 45.1 & 32.4 & & \\
c5665 & 28.8 & 30.5 & 40.2 & 27.6 & 35.2 & \\
c7198 & 1.9 & 17.3 & 45.5 & 2.9 & 32.7 & 29.8 \\
neo-X chromosome arm & a52 & a386 & a1185 & a1350 & a1953 & c29 \\
a386 & 27.3 & & & & & \\
a1185 & 33.0 & 37.8 & & & & \\
a1350 & 39.2 & 45.7 & 17.3 & & & \\
a1953 & 26.8 & 32.4 & 44.8 & 52.0 & & \\
c29 & 46.9 & 42.6 & 51.6 & 46.5 & 30.6 & \\
c3242 & 43.8 & 45.1 & 20.0 & 10.8 & 53.5 & 50.5 \\
\hline
\end{tabular}

(a28 and a708) on the second chromosome and four (a1350, a386, a52, and c29) on the neo-X chromosome arm were previously determined (Chang et al. 2008; Chang 2011). Therefore, together with those markers with known locations and recombination rates (Table 2), a rough map was obtained and shown in Figures 1 and 2. In Figure 1, the marker a28 is located near the breakpoint $D_{5}$ of $\operatorname{In}(2 L) B_{1} D_{5}$ inversion (i.e., on the basal of $2 \mathrm{~L}$ ) and a708 is on the basal of $2 \mathrm{R}$ (Chang et al. 2008; Chang 2011). The marker A185D is probably located at the distal end of $2 \mathrm{R}$ arm due to the equal or over 50-cM distance with all other six markers. The markers c5237 and c5665 are likely located on the distal end of $2 \mathrm{~L}$ arm, and c5237 may be located more distal than c5665 due to higher recombination rates between them and a708 (i.e., between c5237 and a708 is 39.0; between c5665 and a708 is 30.5) than those between them and a28 (i.e., between c5237 and a28 is 33.7; between c5665 and a28 is 28.8). The extremely low pair-wise recombination rates among c4081, c7198, and a28 (Table 2) indicate that they should be regarded as one unit which may be located near or inside the inversion. However, their locations cannot be determined due to heterozygous $\operatorname{In}(2 L) B_{1} D_{5}$ in $\mathrm{F}_{1}$ (Figure 1). Figure 2 shows the markers on the neo-X chromosome arm. There are four markers with known locations including a1350 located near the centromere, a386 and a52 located in the middle of the chromosome, and c29 located on the tip (Chang et al. 2008). Based on the recombination rates (Table 2), we estimated the relative locations for other markers, a1185, a1953, and c3242, as shown in Figure 2. The 14 markers, except 3 related to the $\operatorname{In}(2 L) B_{1} D_{5}$, are scattered on those chromosome arms.

The association between genotypes (i.e., $P P$ and $S S$ ) and parthenogenetic capability (i.e., with and without parthenogenetically produced offspring) was proceeded. As shown in Figure 3, six (a28, a708, c4081, c5237, c5665, and c7198) of the seven markers on the second chromosome showed statistically significant association (Fisher's exact test, $p<0.01$ for $c 5237$ and $p<0.001$ for other five markers), whereas none of the seven markers on the neo-X chromosome arm showed significant association (Fisher's exact test, $p>0.05$ ). High linkage disequilibrium values appeared among all six markers strongly associated with parthenogenetic capability (Table 3). Among these markers, a28 was always related to parthenogenetic capability in those pair-wise comparisons (Table 4). Small amount of recombinants among markers, a708, c5237, and c5665 showed significant difference from a28 (Fisher's exact test, $p<0.01$, Table 4). However, the amount of recombinants was too small to discriminate the contribution among a28, c4081, and c7198. Due to the crossing-over inhibition caused by the heterozygous inversion, we may infer that loci involved in parthenogenetic capability are associated with In $(2 L) B_{1} D_{5}$.

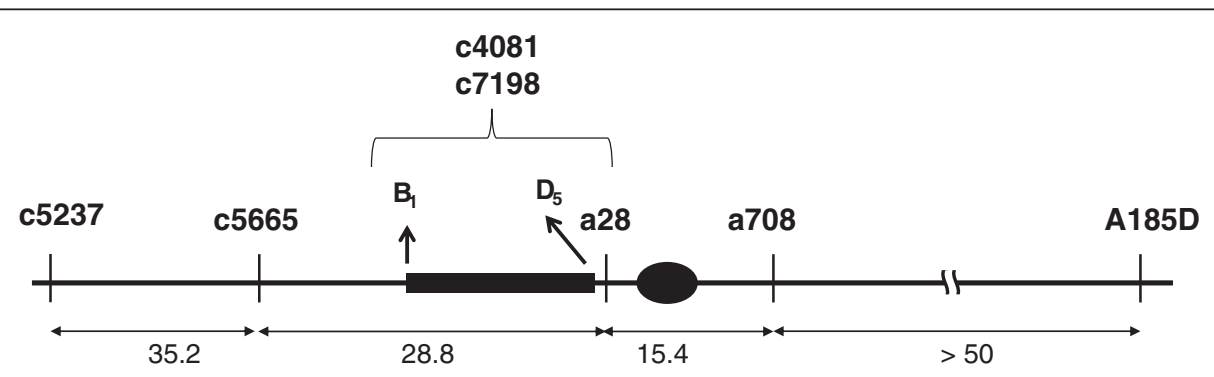

Figure 1 Locations of seven markers on the second chromosome. The genetic distances are shown by percentage of recombination rate. The locations of a28 and a708 were revealed by in situ hybridization (Chang et al. 2008; Chang 2011). The recombination rate statistically indistinguishable from $50 \%$ was marked as $>50 \mathrm{cM}$. Black horizontal line shows the second chromosome, and a black oval dot represents the centromere. The black bar indicates $\ln (2 L) B_{1} D_{5}$ region and $D_{5}$ is near the centromere. Two markers $c 4081$ and $c 7198$ may be located near or inside the inversion (see text). 


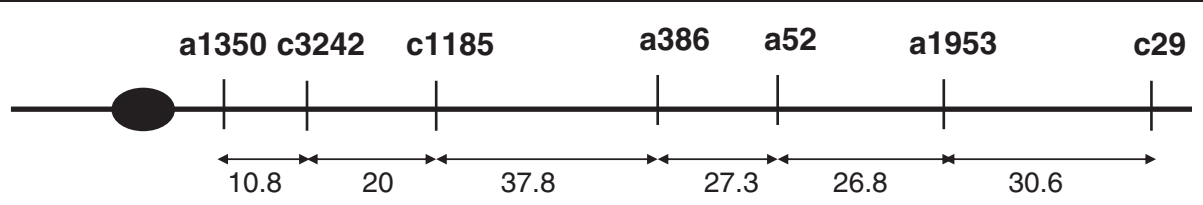

Figure 2 Locations of seven markers on the neo-X chromosome arm. The genetic distances are shown by percentage of recombination rate. The locations of a1350, a386, a52, and c29 were revealed by in situ hybridization (Chang et al. 2008). Black horizontal line shows the neo-X chromosome arm, and a black oval dot represents the centromere.

The distribution of quantitative parthenogenetic fecundity loci

The fecundity of $F_{2}$ varied from 1 to 59 (in average $16.7 \pm$ 16.2) and showed a continuous distribution as expectation of a quantitative trait (Figure 4). The significant correlation between the fecundity and the number of homozygous markers (ANOVA test, $p<0.001$ ) is shown in Figure 5. Through jackknifing, the significant correlation remains no matter which marker was omitted from the re-sampling which indicates no single major gene influencing fecundity.

\section{Discussion}

The facultative parthenogenesis, occasional parthenogenesis with primarily sexual reproduction, is not unusual in insect. It is found in at least ten insect orders (Simon et al. 2003). The success of parthenogenesis is in general dependent on parthenogenetic capability as well as fecundity. In the present study, we performed genetic analysis using 14 molecular markers to reveal that parthenogenetic capability is associated with a limited number of gene loci on 2L chromosome, whereas parthenogenetic fecundity is influenced by numerous quantitative loci distributed on whole genome.

In order to locate the gene for parthenogenetic capability, sexual strains were checked for their parthenogenetic capability. Since females from \#55.1 did not perform parthenogenesis, this strain was chosen to cross with KKU119, and their $F_{2}$ offspring parthenogenetically produced by $F_{1}$ were used for the genetic analysis of parthenogenesis. An advantage of adopting this strain is that their parthenogenetic capability and fecundity must be contributed by KKU119, which has been cultured for 370 generations by parthenogenesis. Their parthenogenetic capability gene(s) should have high or even fixed frequency. Although crossing between strains \#55.1 and KKU119 produces heterozygous $\operatorname{In}(2 L) B_{1} D_{5} \mathrm{~F}_{1}$ and invalidates mapping on a portion of the $2 \mathrm{~L}$ arm, other chromosome regions covered by scattered markers are not influenced. After crossing, all sexually reproduced $F_{1}$ have the same genotypes, but their parthenogenetic $F_{2}$ may have different

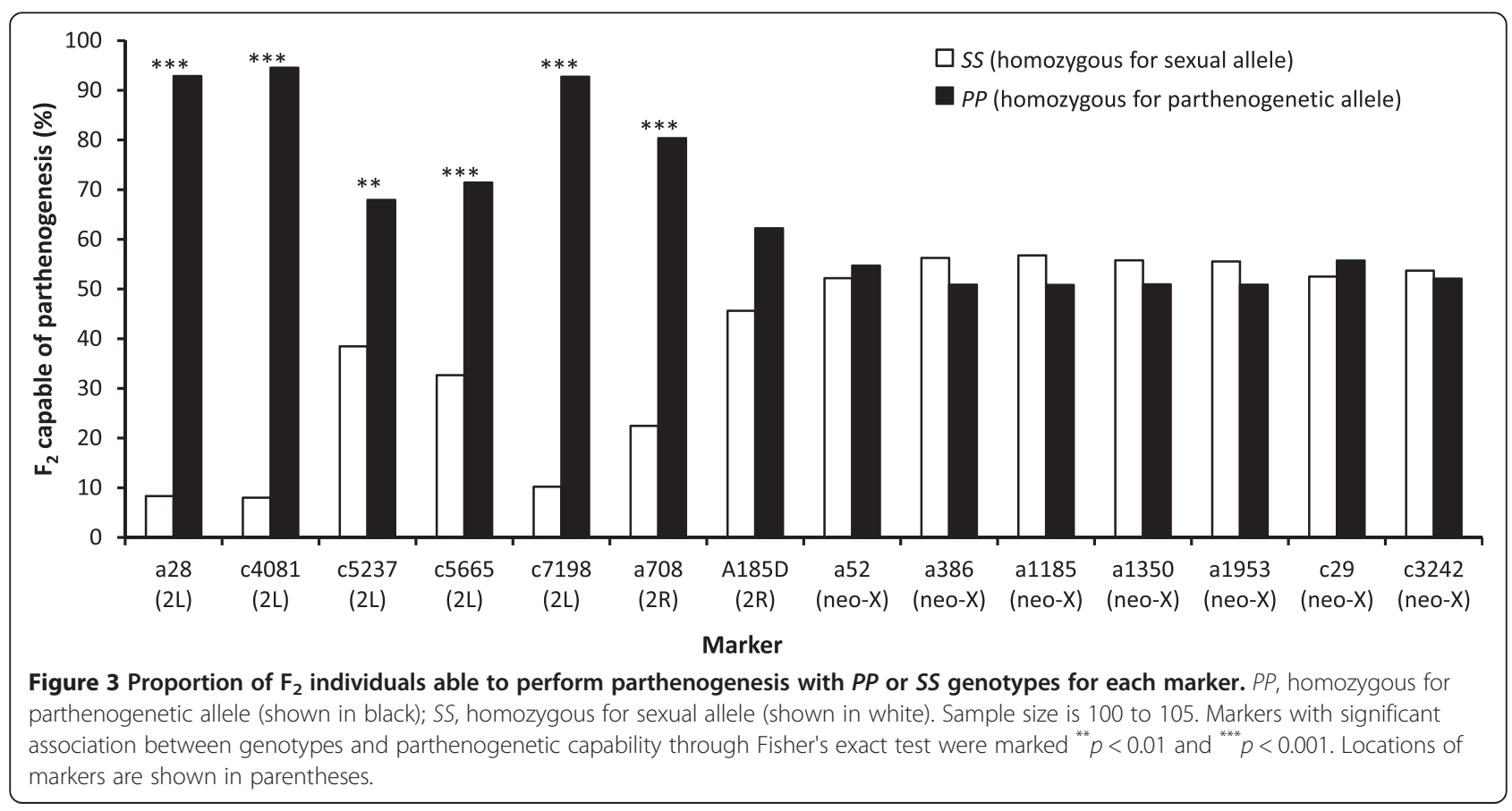


Table 3 Linkage disequilibrium $\left(D^{\prime}\right)$ for markers shown to be highly associated with parthenogenetic capability

\begin{tabular}{llllll}
\hline & a708 & c4081 & c5237 & c5665 & c7198 \\
\hline a28 & 0.93 & 1.00 & 0.59 & 0.71 & 1.00 \\
a708 & & 0.92 & 0.42 & 0.68 & 0.91 \\
c4081 & & & 0.63 & 0.74 & 1.00 \\
c5237 & & & & 0.54 & 0.61 \\
c5665 & & & & & 0.69 \\
\hline
\end{tabular}

genotypes via recombination. Therefore, those $\mathrm{F}_{2}$ were subjected to investigate the association between genotypes and parthenogenetic capability.

About half of recombinant homozygous $\mathrm{F}_{2}$ were unable to do parthenogenesis, which corresponds to the about half which did not acquire the capability allele from the parthenogenetic strain (i.e., ranging from 39\% to $56 \%$ for 14 markers). On the contrary, the other half of them able to do parthenogenesis may carry the parthenogenetic allele. These results implicate that there is only one genetic element responsible for parthenogenetic capability. Among all markers, the three markers, a28, c4081, and c7198, were most strongly associated with parthenogenetic capability. However, due to crossing-over inhibition of the heterozygous $\operatorname{In}(2 L) B_{1} D_{5}$, they were treated as one genetic element contributing to parthenogenesis. In other words, the capability gene may be located very close to $\operatorname{In}(2 L) B_{1} D_{5}$. The only marker on the second chromosome, A185D, which did not associate with parthenogenesis is located over $50 \mathrm{cM}$ apart from the other six markers therefore assorted independently just like markers on neo-X chromosome arm. To sum up, we infer that the parthenogenetic capability gene or gene cluster is located on $2 \mathrm{~L}$ arm and is strongly associated with $\operatorname{In}(2 L)$ $B_{1} D_{5}$. However, due to suppressed recombination rates on

Table 4 Number of recombinant $F_{2}$ categorized into genotypes and whether or not produced $F_{3}$ offspring parthenogenetically

\begin{tabular}{|c|c|c|c|c|}
\hline \multirow{2}{*}{$\begin{array}{l}\text { Pair of } \\
\text { markers with } \\
\text { recombination }\end{array}$} & \multirow[t]{2}{*}{ Genotypes } & \multicolumn{2}{|c|}{ Number of $F_{2}$} & \multirow{2}{*}{$\begin{array}{l}p \\
\text { value }^{a}\end{array}$} \\
\hline & & Without $F_{3}$ & With $F_{3}$ & \\
\hline \multirow[t]{2}{*}{ a28, c5237 } & $S S, P P$ & 15 & 1 & $<0.001$ \\
\hline & $P P, S S$ & 2 & 17 & \\
\hline \multirow[t]{2}{*}{ a28, c5665 } & $S S, P P$ & 15 & 0 & $<0.001$ \\
\hline & $P P, S S$ & 3 & 12 & \\
\hline \multirow[t]{2}{*}{ a28, a708 } & $S S, P P$ & 8 & 0 & $<0.01$ \\
\hline & $P P, S S$ & 1 & 7 & \\
\hline \multirow[t]{2}{*}{ a28, c7198 } & $S S, P P$ & 1 & 0 & NA \\
\hline & $P P, S S$ & 0 & 1 & \\
\hline \multirow[t]{2}{*}{ a28, c4081 } & $S S, P P$ & 0 & 0 & NA \\
\hline & $P P, S S$ & 1 & 0 & \\
\hline
\end{tabular}

aased on Fisher's exact test. NA, not applicable.

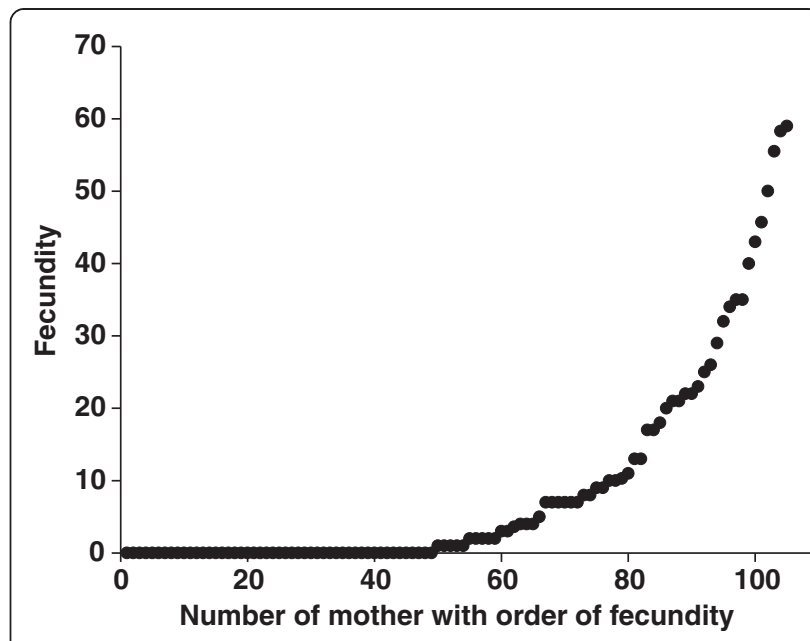

Figure 4 Fecundity of $F_{2}$ through parthenogenesis. Each dot represents the fecundity of each $F_{2}$ female and ordered by fecundity. Sample size is 105.

$2 \mathrm{~L}$ arm in heterozygous $\mathrm{F}_{1}$, the precise location of the major gene(s) was difficult to be mapped by current genetic analysis. To that purpose, it is essential to find another sexual strain which is unable to perform parthenogenesis while carries the same chromosome arrangement of $2 \mathrm{~L}$ as that of the parthenogenetic strain for detailed mapping of the major gene(s).

Since the major gene or gene cluster is highly linked to $\operatorname{In}(2 L) B_{1} D_{5}$ inversion, the high $\operatorname{In}(2 L) B_{1} D_{5}$ heterozygosity may be one reason for the preservation of parthenogenetic capability in Drosophila albomicans. It was reported that Drosophila mercatorum from natural populations which carried high heterozygosity are more capable of parthenogenesis than those from sexual inbreeding which carried high homozygosity (Templeton et al. 1976a), so it may suggest that the high heterozygosity plays an important role in the maintenance of parthenogenesis. A Drosophila albomicans sexual strain \#56.1 originating from Wulai population, which has the closest phylogenetic relationship to Japan population (Ohsako et al. 1994), was able to do parthenogenesis in spite of strong genetic drift and lacking selection for parthenogenesis with 40-year long-term sexual reproduction culture. The high $\operatorname{In}(2 L) B_{1} D_{5}$ heterozygosity in \#56.1 probably preserved parthenogenetic capability gene in the laboratory strain. In natural populations, high $\operatorname{In}(2 L) B_{1} D_{5}$ heterozygosity was observed in winter in Wulai populations (Chang et al. 1987; Yang et al. 2002) and in the Japan population (Ohsako et al. 1994). Inversion heterosis may be responsible for the maintenance of parthenogenetic capability alleles in a population; however, inversion may not have direct relationship with the gene.

Parthenogenetic fecundity may be contributed by many loci widely distributed on whole genome. The parthenogenetic fecundity of $\mathrm{F}_{2}$ was shown as a quantitative trait, 


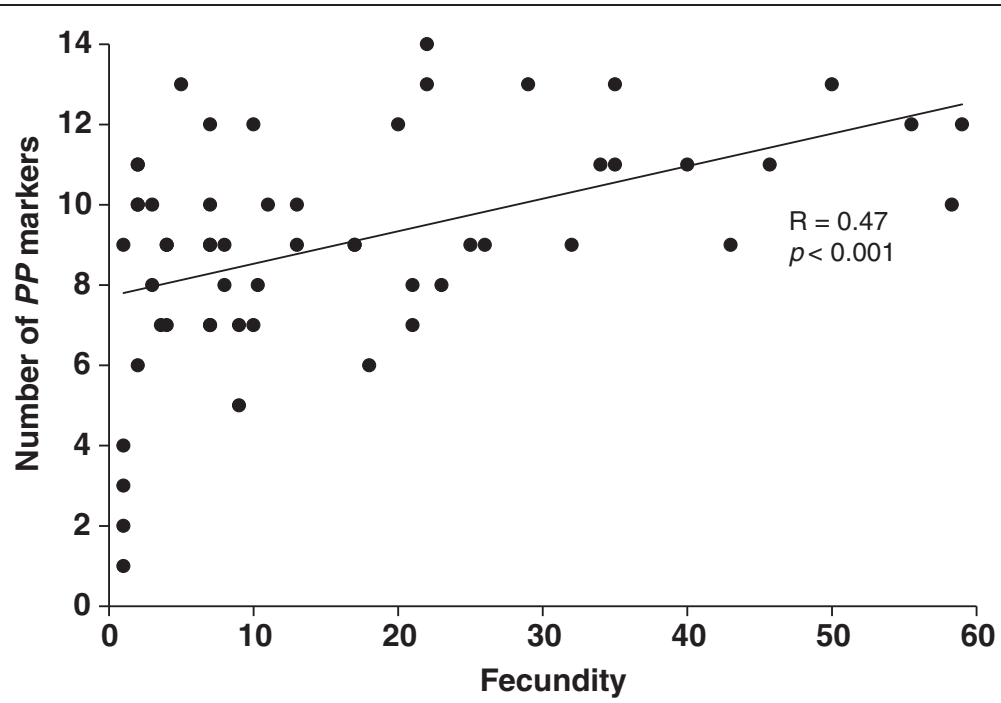

Figure 5 The correlation between the fecundity and the number of $P P$ markers. The $x$-axis is the fecundity of each $F_{2}$, and the $y$-axis is the number of $P P$ (homozygous for parthenogenetic alleles) markers for each $F_{2}$. Sample size is 56. ANOVA test, $p<0.001$.

suggesting that there may have many loci with additive effect influencing fecundity. The significant correlation between the fecundity and the number of homozygous markers (ANOVA test, $p<0.001$ ) suggests that the individuals with more $P P$ markers may tend to have higher fecundity. That is, there are many loci with additive effect influencing parthenogenetic fecundity. The consistent significant correlation through jackknifing supports the quantitative trait model (i.e., many loci with small effect) and numerous alleles regulate the parthenogenetic fecundity. Identification of genes involved in parthenogenetic fecundity warrants our further investigation. In Drosophila mercatorum, the 'coadapted genome' for parthenogenesis was suggested through the decreasing fecundity with higher perturbation level, through recombinant lines generated by crossing different parthenogenetic strains (Templeton et al. 1976b), and the coadaptation was formed effectively under the selection for parthenogenesis (Templeton 1979). Therefore, the additive effect for parthenogenetic fecundity is likely to respond to artificial selection for parthenogenesis (Ohsako and Fuyama 1995), and it might be coadapted with the gene for parthenogenetic capability.

\section{Conclusions}

In Drosophila albomicans, parthenogenetic capability is regulated by one genetic element located on $2 \mathrm{~L}$ arm, and the high $\operatorname{In}(2 L) B_{1} D_{5}$ heterozygosity may play a role in preserving parthenogenetic capability since the genetic element responsible for parthenogenetic capability is closely associated with $\operatorname{In}(2 L) B_{1} D_{5}$. Moreover, parthenogenetic fecundity shown as a quantitative trait by its continuous distribution is regulated by several additive loci, and through jackknifing, there is no highly influential one among the 14 markers. Therefore, we may infer that parthenogenetic fecundity is related to many genes in the genome including $2 \mathrm{R}, 2 \mathrm{~L}$, and neo-X chromosome arms.

\section{Additional file}

Additional file 1: The fecundity and $\mathbf{1 4}$ molecular marker
genotypes of $\mathbf{1 0 5} \mathbf{F}_{\mathbf{2}}$. The additional file contains the fecundity which is
the number of offspring parthenogenetically reproduced by $F_{2}$ and the
genotypes of 14 molecular markers. Each row represents one $F_{2}$
individual with their own number. $P$ represents the allele from the
parthenogenetic strain, KKU119, and $S$ represents the allele from the
sexual strain, \#55.1. NA means the data was not obtained successfully.

Competing interests

The authors declare that they have no competing interests.

\section{Authors' contributions}

$\mathrm{CC}$ and $\mathrm{HC}$ designed the study and wrote the manuscript. CC carried out the laboratory work and analyzed the data. Both authors read and approved the final manuscript.

\section{Acknowledgements}

We are grateful to Dr. Y. Fuyama for generously providing the parthenogenetic Drosophila albomicans strain, and we also thank the Sequencing Core Facility, Scientific Instrument Center at Academia Sinica for DNA sequencing. This work was partly funded by the grant (NSC100-2815-C002-142B) of National Science Council (Taiwan) for undergraduate student to CC.

Received: 25 February 2014 Accepted: 11 June 2014

Published online: 03 July 2014

\section{References}

Annest JL, Templeton AR (1978) Genetic recombination and clonal selection in Drosophila mercatorum. Genetics 89:193-210

Carson HL (1967) Selection for parthenogenesis in Drosophila mercatorum. Genetics 55:157-171 
Chang CH (2011) Early-stage evolution of the neo-Y chromosomes in Drosophila albomicans, Master thesis, Graduate Institute of Ecology and Evolutionary Biology. National Taiwan University, Taipei, Taiwan

Chang H, Chang SH, Lin FJ (1987) Effects of climatic factors on the heterozygosity of $\ln (2 L) B_{1} D_{5}$ in Drosophila albomicans. B I Zool Acad Sinica 26:39-45

Chang TP, Tsai TH, Chang H (2008) Fusions of Muller's elements during chromosome evolution of Drosophila albomicans. Zool Stud 47:574-584

Fuyama Y (1986) Genetics of parthenogenesis in Drosophila melanogaster. II. Characterization of a gynogenetically reproducing strain. Genetics 114:495-509

Hurst LD, Peck JR (1996) Recent advances in understanding of the evolution and maintenance of sex. Trends Ecol Evol 11:46-52

Lewontin RC (1964) The interaction of selection and linkage. I. General considerations; heterotic models. Genetics 49:49-67

Markow TA (2013) Parents without partners: Drosophila as a model for understanding the mechanisms and evolution of parthenogenesis. G3-Genes Genom Genet 3:757-762

Matsuda M, Tobari YN (2004) Genetic analyses of several Drosophila ananassae-complex species show a low-frequency major gene for parthenogenesis that maps to chromosome 2. Genet Res 83:83-89

Maynard Smith J (1978) The evolution of sex. Cambridge University Press, New York

Ohsako T, Fuyama Y (1995) Drosophila albomicans, a new member of parthenogenetic Drosophila. Dros Inf Serv 76:150

Ohsako T, Aotsuka T, Kitagawa O (1994) The origins of the Japanese mainland population of Drosophila albomicans. Jpn I Genet 69:183-194

Schwander T, Vuilleumier S, Dubman J, Crespi BJ (2010) Positive feedback in the transition from sexual reproduction to parthenogenesis. Proc Biol Sci 277:1435-1442

Simon JC, Delmotte F, Rispe C, Crease T (2003) Phylogenetic relationships between parthenogens and their sexual relatives: the possible routes to parthenogenesis in animals. Biol J Linn Soc 79:151-163

Stalker HD (1954) Parthenogenesis in Drosophila. Genetics 39:4-34

Suomalainen E (1950) Parthenogenesis in animals. Adv Genet 3:193-253

Templeton AR (1979) The unit of selection in Drosophila mercatorum. II. Genetic revolution and the origin of coadapted genomes in parthenogenetic strains. Genetics 92:1265-1282

Templeton AR (1983) Nature and experimental parthenogenesis. In: The genetics and biology of Drosophila, vol 3c. Academic, New York, pp 343-398

Templeton AR, Carson HL, Sing CF (1976a) The population genetics of parthenogenetic strains of Drosophila mercatorium. II The capacity for parthenogenesis in a natural, bisexual population. Genetics 82:527-542

Templeton AR, Sing CF, Brokaw B (1976b) The unit of selection in Drosophila mercatorum. I. The interaction of selection and meiosis in parthenogenetic strains. Genetics 82:349-376

Yang YY, Lin FJ, Chang H (2002) Comparison of recessive lethal accumulation in inversion-bearing and inversion-free chromosomes in Drosophila. Zool Stud 41:271-282

doi:10.1186/s40555-014-0035-1

Cite this article as: Chang and Chang: Genetic analysis of parthenogenetic capability and fecundity in Drosophila albomicans. Zoological Studies 2014 53:35.

\section{Submit your manuscript to a SpringerOpen ${ }^{\circ}$ journal and benefit from:}

- Convenient online submission

- Rigorous peer review

- Immediate publication on acceptance

- Open access: articles freely available online

- High visibility within the field

- Retaining the copyright to your article

Submit your next manuscript at $\gg$ springeropen.com 